\title{
Time-dependent analysis of a fiber-optic passive-loop resonator
}

\author{
B. Crosignani* and A. Yariv \\ California Institute of Technology, Pasadena, California 91125 \\ P. Di Porto \\ Dipartimento di Fisica, Università dell'Aquila, L'Aquila, Italy
}

Received September 20, 1985; accepted January 31, 1986

\begin{abstract}
A time-dependent analysis of an all-single-mode fiber-optic resonator is presented in which the input field is allowed to exhibit an arbitrary dependence on time. In particular, the transmissivity of the resonator is evaluated for an input field possessing an arbitrary temporal coherence, which allows one to consider the role of the source coherence time as compared with the fiber time delay.
\end{abstract}

A passive-loop resonator can be constructed by means of a polarization-preserving monomode optical fiber and of a low-loss single-mode-fiber directional coupler, as illustrated in Fig. 1. An input field

$$
E_{1}(z, t)=\exp (i \beta z-i \omega t) \epsilon_{1}(t)
$$

launched into the coupler at the input port 1 will partially emerge at port 3 , from which it is guided along the fiber loop and reaches port 2 and will be partially coupled to the output port 4 . The field at port 2 will couple partially to port 4 and partially to port 3 , thus continuing to circulate in the loop.

The characteristics of this all-fiber ring resonator have been investigated both theoretically and experimentally ${ }^{1,2}$ in a completely coherent case in which the coherence time $t_{c}$ of the field is implicitly assumed significantly to exceed $\tau=L / V$, where $L$ is the fiber loop length and $V=(\mathrm{d} \beta / \mathrm{d} \omega)^{-1}$ is the group velocity of the propagating mode. More recently, an analysis of the performance characteristics of the resonator in terms of the light-source coherence was carried out ${ }^{3}$ in which the exciting source was assumed to be an amplitude-stabilized single-mode laser field

$$
E(t)=E_{0} \exp [-i \omega t+i \phi(t)]
$$

with a correlation time $\tau^{*}$ of the instantaneous frequency-correlation function $\left\langle\dot{\phi}(t) \dot{\phi}\left(t^{\prime}\right)\right\rangle$ much shorter than $\tau$.

In this Letter we report on the case in which $\epsilon_{1}(t)$ exhibits an arbitrary time dependence (as long as the bandwidth of the input field satisfies the relation $\delta \omega / \omega$ $\ll 1$ and the fiber chromatic dispersion can be neglected) and derive a general expression for the resonator transmissivity $T$ in terms of the autocorrelation function

$$
G(\tau)=\frac{\left\langle\epsilon_{1}(t) \epsilon_{1}^{*}(t-\tau)\right\rangle}{\left\langle\left|\epsilon_{1}(t)\right|^{2}\right\rangle}
$$

of the input field. This analysis finds its practical justification in the fact that, as the length $L$ of the loop increases, $\tau$ can become comparable with $t_{c}$.

The behavior of the field inside the resonator can easily be obtained by relying on the pertinent geometry sketched in Fig. 1. More precisely, after indicating by $\epsilon_{3}(t)$ and $\epsilon_{4}(t)$ the complex amplitudes of the fields at ports 3 and 4 , one immediately gets

$$
\begin{aligned}
\epsilon_{3}(t)= & \left(1-\gamma_{0}\right)^{1 / 2}\left[(1-k)^{1 / 2} \epsilon_{1}(t)+i k^{1 / 2}\right. \\
& \left.\times \exp \left(-\alpha_{0} L+i \beta L\right) \epsilon_{3}(t-\tau)\right], \\
\epsilon_{4}(t)= & \left(1-\gamma_{0}\right)^{1 / 2}\left[i k^{1 / 2} \epsilon_{1}(t)+(1-k)^{1 / 2}\right. \\
& \left.\times \exp \left(-\alpha_{0} L+i \beta L\right) \epsilon_{3}(t-\tau)\right],
\end{aligned}
$$

where $k$ is the (intensity) coupling coefficient, $\gamma_{0}=$ $\left(\left|\epsilon_{1}\right|^{2}+\left|\epsilon_{2}\right|^{2}-\left|\epsilon_{3}\right|^{2}-\left|\epsilon_{4}\right|^{2}\right) /\left(\left|\epsilon_{1}\right|^{2}+\left|\epsilon_{2}\right|^{2}\right)$ is the fractional coupler intensity loss, and we have used the relation

$$
\epsilon_{2}(t)=\exp \left(-\alpha_{0} L+i \beta L\right) \epsilon_{3}(t-\tau),
$$

$\alpha_{0}$ being the amplitude-attenuation coefficient of the fiber. By eliminating $\epsilon_{3}$ from the set of Eqs. (4), one finds the following relation between $\epsilon_{4}$ and $\epsilon_{1}$ :

$$
\epsilon_{4}(t)-\lambda \epsilon_{4}(t-\tau)=a \epsilon_{1}(t)+b \epsilon_{1}(t-\tau),
$$

where $\lambda \equiv i\left(1-\gamma_{0}\right)^{1 / 2} k^{1 / 2} \exp \left(-\alpha_{0} L+i \beta L\right), a \equiv i(1-$ $\left.\gamma_{0}\right)^{1 / 2} k^{1 / 2}$, and $b \equiv\left(1-\gamma_{0}\right) \exp \left(-\alpha_{0} L+i \beta L\right)$.

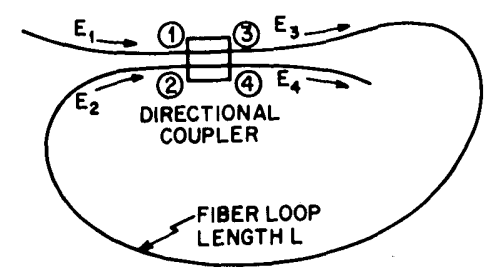

Fig. 1. Fiber-optic passive loop resonator. 
Equation (6) has the form of a Fredholm linear integral of the second kind,

$$
\epsilon_{4}(t)-\lambda \int K\left(t, t^{\prime}\right) \epsilon_{4}\left(t^{\prime}\right) \mathrm{d} t^{\prime}=F(t),
$$

where $K\left(t, t^{\prime}\right) \equiv \delta\left[t^{\prime}-(t-\tau)\right](\delta$ being the Dirac delta function) and $F(t) \equiv a \epsilon_{1}(t)+b \epsilon_{1}(t-\tau)$. This is solved using the standard iterative procedure ${ }^{4}$ to get

$$
\epsilon_{4}(t)=a \epsilon_{1}(t)+h \sum_{n=0}^{\infty} \lambda^{n} \epsilon_{1}[t-(n+1) \tau],
$$

where $h=b+\lambda a$.

Since the input field is random, the signficant quantity to evaluate is the power $\left\langle\left|\epsilon_{4}(t)\right|^{2}\right\rangle$, where \langle\rangle denotes the ensemble average (or the time average in an interval long compared with $t_{c}$ in a stationary situation). According to Eq. (8) one has

$$
\begin{aligned}
\left\langle\left|\epsilon_{4}(t)\right|^{2}\right\rangle= & |a|^{2}\left\langle\left|\epsilon_{1}(t)\right|^{2}\right\rangle+a h^{*} \\
& \times \sum_{n=0}^{\infty} \lambda^{*}{ }^{*}\left\langle\epsilon_{1}(t) \epsilon_{1}{ }^{*}[t-(n+1) \tau]\right\rangle \\
& +a^{*} h \sum_{n=0} \lambda^{n}\left\langle\epsilon_{1}^{*}(t) \epsilon_{1}[t-(n+1) \tau]\right\rangle \\
& +|h|^{2} \sum_{n=0}^{\infty} \sum_{m=0}^{\infty} \lambda^{n} \lambda^{*}\left\langle\epsilon_{1}[t-(n+1) \tau]\right. \\
& \left.\times \epsilon_{1}{ }^{*}[t-(m+1) \tau]\right\rangle .
\end{aligned}
$$

One can now regroup the above expression into the various terms $\left\langle\left|\epsilon_{1}(t)\right|^{2}\right\rangle,\left\langle\epsilon_{1}(t) \epsilon_{1} *(t-\tau)\right\rangle, \ldots$, $\left\langle\epsilon_{1}(t) \epsilon_{1}{ }^{*}(t-s \tau)\right\rangle, \ldots$ (note that, owing to the assumption of stationarity, these correlation functions depend on the differences between their temporal arguments), a procedure that allows Eq. (9) to be rewritten as

$$
\begin{aligned}
\mid\left\langle\left|\epsilon_{4}(t)\right|^{2}\right\rangle= & \left(|a|^{2}+|h|^{2} \sum_{n=0}^{\infty}|\lambda|^{2 n}\right)\left\langle\left|\epsilon_{1}(t)\right|^{2}\right\rangle \\
& +\left[a h^{*}+a^{*} h+|h|^{2}\left(\sum_{n=0}^{\infty} \lambda^{n} \lambda^{* n+1}\right.\right. \\
& \left.\left.+\sum_{n=0}^{\infty} \lambda^{n+1} \lambda^{* n}\right)\right]\left\langle\epsilon_{1}(t) \epsilon_{1}{ }^{*}(t-\tau)\right\rangle \\
& +\left[a h^{*} \lambda^{*}+a^{*} h \lambda+|h|^{2}\left(\sum_{n=0}^{\infty} \lambda^{n} \lambda^{* n+2}\right.\right. \\
& \left.\left.+\sum_{n=0}^{\infty} \lambda^{n+2} \lambda^{* n}\right)\right]\left\langle\epsilon_{1}(t) \epsilon_{1}{ }^{*}(t-2 \tau)\right\rangle+\ldots
\end{aligned}
$$

All the series appearing in Eq. (10) are geometric and can thus be summed so that one can finally express the transmissivity $T$ of the fiber resonator as a sum of terms of decreasing magnitude:

$$
\begin{aligned}
T= & \frac{\left\langle\left|\epsilon_{4}(t)\right|^{2}\right\rangle}{\left\langle\left|\epsilon_{1}(t)\right|^{2}\right\rangle}=\left[|a|^{2}+|h|^{2} /\left(1-|\lambda|^{2}\right)\right] \\
& \times\left\{G(0)+\sum_{n=0}^{\infty}\left(\lambda^{n+1}+\lambda^{* n+1}\right) G[(n+1) \tau]\right\} \\
& +\sum_{n=0}^{\infty}\left(a b^{*} \lambda^{* n}+a^{*} b \lambda^{n}\right) G[(n+1) \tau] .
\end{aligned}
$$

In the two limiting cases of either very short or very long coherence time [where one has, respectively, $G(n \tau)=0$ for $n \geqq 1$ and $G(n \tau)=1$ ], Eq. (11) becomes, after introducing $M \equiv\left(1-\gamma_{0}\right) \exp \left(-2 \alpha_{0} L\right)$,

$$
\begin{aligned}
T\left(t_{c} \ll \tau\right)= & |a|^{2}+|h|^{2} /\left(1-|\lambda|^{2}\right)=\left(1-\gamma_{0}\right) \\
& \times[1-(1-k)(1-M) /(1-k M)], \\
T\left(t_{c} \gg \tau\right)= & |a+b|^{2} /|1-\lambda|^{2}=\left(1-\gamma_{0}\right) \\
& \times \frac{k+M+2 k^{1 / 2} M^{1 / 2} \sin (\beta L)}{1+k M+2 k^{1 / 2} M^{1 / 2} \sin (\beta L)} .
\end{aligned}
$$

Equation (11), in particular, allows one to recover the expression for $T$ derived in Ref. 3 for a single-mode amplitude-stabilized laser field with a Lorentzian spectrum for which $G(\tau)=\exp (-\Delta \omega \tau)$ and, consequently, $G(n \tau)=[G(\tau)]^{n} \equiv \delta^{n}$. In fact, inserting this relation into Eq. (11) facilitates the summation of the series with the following result:

$$
\begin{aligned}
T= & \left\{\left(1-|\lambda|^{2} \delta^{2}\right)\left(|a|^{2}+|b|^{2}\right)+a b^{*}\left[\delta\left(1-|\lambda|^{2}\right)\right.\right. \\
& \left.+\lambda\left(1-\delta^{2}\right)\right]+a^{*} b\left[\delta\left(1-|\lambda|^{2}\right)\right. \\
& \left.\left.+\lambda^{*}\left(1-\delta^{2}\right)\right]\right\} /|1-\lambda \delta|^{2}\left(1-|\lambda|^{2}\right),
\end{aligned}
$$

or, with the definitions

$$
\begin{aligned}
F & \equiv(1-k)(1-M)\left(1-k M \delta^{2}\right) /(1-k M)\left(1+\delta k^{1 / 2} M^{1 / 2}\right)^{2}, \\
H & \equiv 4 \delta k^{1 / 2} M^{1 / 2} /\left(1+\delta k^{1 / 2} M^{1 / 2}\right)^{2}, \quad \theta=\beta L-\pi / 2,
\end{aligned}
$$

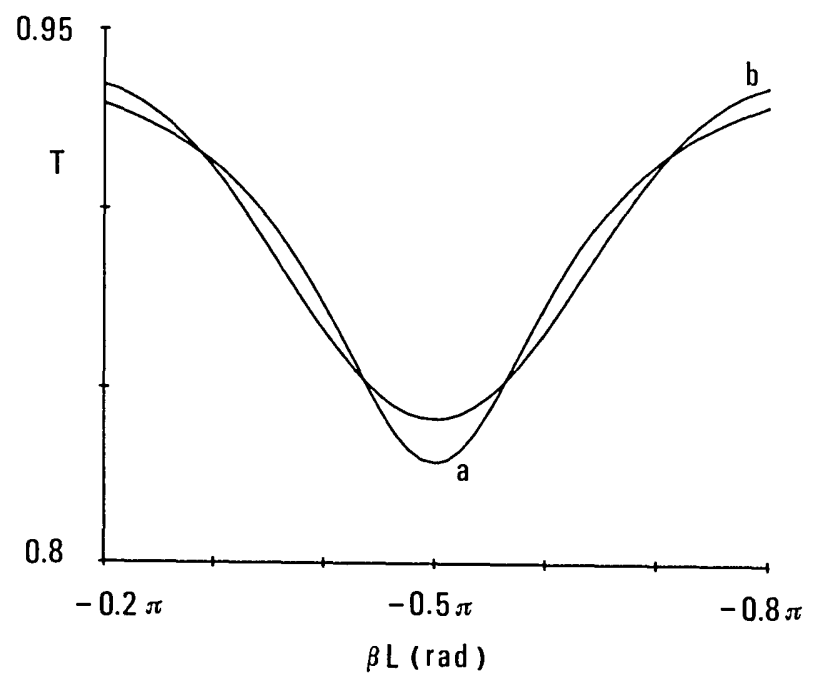

Fig. 2. Transmissivity $T$ as a function of $\beta L$ for $k=0.95, \gamma_{0}$ $=0.05, M \simeq 1-\gamma_{0}$, and $\delta=0.7$. Curve a, exact solution; curve b, approximate solution $(n=5)$. 


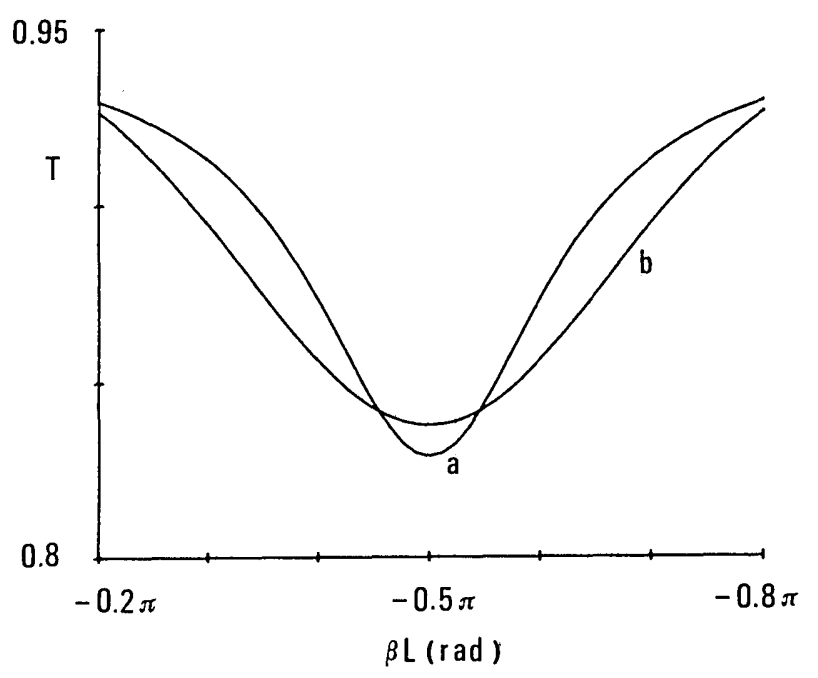

Fig. 3. Transmissivity $\dot{T}$ as a function of $\beta L$ for $k=0.95, \gamma_{0}$ $=0.05, M \simeq 1-\gamma_{0}$, and $\delta=0.7$. Curve a, $G(\tau)=\exp (-\Delta \omega \tau)$; curve b, $G(\tau)=\exp \left[-(\Delta \omega \tau)^{2}\right]$.

$$
T=\left(1-\gamma_{0}\right)\left[1-\frac{F}{1-H \sin ^{2}(\theta / 2)}\right] \text {. }
$$

The general expression furnished by Eq. (11) can also be rewritten as

$$
\begin{aligned}
T= & \left(1-\gamma_{0}\right)\left(1-\frac{(1-k)(1-M)}{1-k M}\left\{1+2 \sum_{n=0}^{\infty}\right.\right. \\
& \times(k M)^{(n+1) / 2} G[(n+1) \tau] \\
& \times \cos [(n+1)(\beta L+\pi / 2)]\}),
\end{aligned}
$$

which yields the resonance condition $\beta L=2 \pi q-\pi / 2$, $q$ being any integer. The sharpness of the resonance (that is, the finesse of the loop resonator) is a complicated function of the degree of coherence that has to be numerically evaluated by truncating the series $[k M$ $\langle 1, G(n \tau)<1]$.

To estimate the rapidity of the series convergence, we have plotted in Fig. 2 the exact behavior of $T$ corresponding to Eq. (15) (curve a) and that obtained by truncating the series [Eq. (16)] with $G(n \tau)=\delta^{n}$ at the $n=5$ term (curve b). The agreement is reasonable for the given choice of parameters and is excellent for $n=10$.

Finally, in order to present the influence of the shape of the correlation function $G(\tau)$ (or, equivalently, of the source spectral behavior) on the performance of the resonator, we compare in Fig. 3 the transmissivities for $G(\tau)=\exp (-\Delta \omega \tau)=\delta$ and $G(\tau)=$ $\exp \left[-(\Delta \omega \tau)^{2}\right]=\exp \left(-\ln ^{2} \delta\right)$ with a common value of $\delta$ obtained by truncating the series.at $n=20$. In physical terms, these correspond to an amplitude-stabilized laser field with an instantaneous frequency-correlation time $\tau^{*}$ [see Eq. (2)] much shorter and much longer, respectively, than $\tau$.

This research was supported by the National Science Foundation, the U.S. Office of Naval Research, and NATO Research Grant 388/84. B. Crosignani and P. Di Porto are also with Fondazione Ugo Bordoni.

* Permanent address, Dipartimento di Fisica, Università di Roma "La Sapienza," Roma, Italy.

\section{References}

1. L. F. Stokes, M. Chodorow, and H. J. Shaw, Opt. Lett. 7, 288 (1982).

2. L. F. Stokes, M. Chodorow, and H. J. Shaw, J. Lightwave Technol. LT-1, 110 (1983).

3. Y. Ohtsuka, J. Lightwave Technol. LT-3, 378 (1985); see also M. Tur, B. Moslehi, and J. W. Goodman, J. Lightwave Technol. LT-3, 20 (1985).

4. See, for example, G. Korn and T. Korn, Mathematical Handbook for Scientists and Engineers (McGraw-Hill, New York, 1961). 